\title{
Relationship between N-terminal pro-B-type natriuretic peptide levels and metabolic syndrome
}

\author{
Yuanyuan Bao ${ }^{1 \#}$, Xiliang Shang ${ }^{2 \#}$, Linuo Zhou ${ }^{1}$, Renming $\mathrm{Hu}^{1}$, Yiming $\mathrm{Li}^{1}$, Wei Ding ${ }^{1}$
}

\begin{abstract}
1Department of Endocrinology and Metabolism, Huashan Hospital, Fudan University, Shanghai, China

2Department of Sports Medicine, Huashan Hospital, Fudan University, Shanghai, China \#These first two authors contributed equally to this work
\end{abstract}

Submitted: 25 October 2010

Accepted: 16 December 2010

Arch Med Sci 2011; 7, 2: 247-256

DOI: $10.5114 /$ aoms.2011.22075

Copyright @ 2011 Termedia \& Banach

\section{Abstract}

Introduction: Previous studies have shown that obese individuals have reduced natriuretic peptide levels. But conflicting data exist on the relation of natriuretic peptide levels to other metabolic risk factors.

Material and methods: We investigated the relationship between plasma $\mathrm{N}$-terminal pro-B-type natriuretic peptide levels (NT-proBNP) and metabolic syndrome (MetS) and metabolic risk factors in 469 patients free of heart failure. Two hundred thirty diagnosed MetS cases and 239 non-MetS cases were included in this study. Echocardiography examinations were performed and left ventricular mass index was calculated according to the Devereux correction formula. NT-proBNP was measured by electrochemiluminescence. The log-transformed NT-proBNP levels were used for abnormal distribution. Multiple linear regression analysis was performed to assess the association between levels of NT-proBNP and metabolic factors. Covariance analysis was used for group comparisons.

Results: Log NT-proBNP levels were independently related to age, gender, body mass index, systolic blood pressure, diastolic blood pressure, fasting blood glucose, triglyceride, high density lipoprotein cholesterol, low density lipoprotein cholesterol, left ventricular mass index and left ventricular ejection fraction in multiple linear regression analysis $(p<0.05)$. Adjusted log NT-proBNP levels were lower in persons with MetS compared with those without MetS $(p<0.05)$. Individuals with hyperlipidaemia, elevated body mass index, diastolic blood pressure and fasting plasma glucose had lower levels of log NT-proBNP than those without MetS $(p<0.05)$.

Conclusions: There is a relationship between metabolic components and lower plasma NT-proBNP concentration. These findings raise the possibility that reduced plasma NT-proBNP levels are a manifestation of MetS, which might possess significant clinical and pathophysiological implications.

Key words: natriuretic peptide, hyperlipidaemia, left ventricular mass index, risk factors.

\section{Introduction}

The metabolic syndrome (MetS) is a worldwide problem, which refers to a constellation of coronary heart disease (CHD) risk factors including obesity and abdominal fat distribution, disorders of glucose and lipid metabolism, and hypertension. The burden of MetS is likely to continue to rise, largely due to decreased physical activity and increases in obesity in our society.

\author{
Corresponding author: \\ Linuo Zhou \\ Department of Endocrinology \\ and Metabolism \\ Huashan Hospital \\ Fudan University \\ Shanghai, China \\ Phone: 86-21-52888143 \\ Fax: 86-21-62496020 \\ E-mail: zhoulinuo@yahoo.cn
}


Obesity is associated with depressed $\mathrm{N}$-terminal pro-B-type natriuretic peptide (NT-proBNP), a cardiac hormone that was recognized as a new and useful biochemical marker for both diagnosis and ruling out of congestive heart failure [1]. Despite the welldocumented association between NT-proBNP levels and obesity, data on relations with other metabolic risk factors are mixed $[2,3]$. Other studies have not found an association between plasma natriuretic peptide levels and hyperlipidaemia [4] or hyperglycaemia [5]. However, Olsen et al. [6] reported an inverse association between NT-proBNP levels and plasma lipids and glucose.

Thus, we sought to elucidate the relations between plasma NT-proBNP levels and metabolic risk factors and the metabolic syndrome in a Chinese study. We hypothesized that plasma NT-proBNP levels would be lower in the presence of the metabolic syndrome or its components.

\section{Material and methods}

\section{Study population}

We retrospectively evaluated the 250 MetS patients and 250 non-MetS cases who were admitted to the department of endocrinology of Huashan Hospital affiliated to Fudan University from July 2005 to August 2009. Participants were excluded for the following reasons: unavailable NT-proBNP levels $(n=20)$, hepatic (total bilirubin level $>5 \mathrm{mg} / \mathrm{dl}$; $n=0$ ) or renal insufficiency (creatinine $>2.0 \mathrm{mg} / \mathrm{dl}$; $n=13)$, a history of heart failure $(n=11)$, and missing covariates $(n=3)$. After these exclusions, 469 subjects ( $94 \%$ of attendees) remained eligible. Written consent was obtained from all patients before the study.

\section{Measurements}

The subjects were interviewed for the documentation of medical histories (hypertension and diabetes) and medication, history of smoking habits (current, former or never), laboratory assessment of cardiovascular disease risk factors, and standardized echocardiographic examination. The body weight of the subjects, wearing light clothing and without their shoes on, was measured with $0.1 \mathrm{~kg}$ precision. Height was measured to the nearest $0.5 \mathrm{~cm}$. Body mass index was calculated as the weight in kilograms divided by the square of height in metres. Systolic and diastolic blood pressure values were the means of two physician-obtained measurements on the left arm of the seated participant. Diabetes was defined by either a history of fasting glucose $\geq 126 \mathrm{mg} / \mathrm{dl}(7.0 \mathrm{mmol} / \mathrm{l})$ or the use of insulin or hypoglycaemic medications.

\section{Laboratory assays}

Peripheral venous blood samples were taken into tubes in the fasting state in all subjects. It was centrifuged at $3000 \mathrm{rpm}$ for $10 \mathrm{~min}$ for plasma separation and immediately used to measure NT-proBNP and other parameters.

Fasting plasma glucose and postprandial blood glucose concentration (glucose oxidase method, GOX0560), serum triglycerides (GPO-PAP method, TGP0560), high density lipoprotein cholesterol and low density lipoprotein cholesterol (IRC method), and serum creatinine (Sarcosine Oxidase-PAP method, S708) of all the patients were estimated on an automated chemistry analyser (Hitachi 7600020, Hitachi High-Technologies Corp, Shanghai, China). Glycated haemoglobin was estimated by high-pressure liquid chromatography using a machine (HLC-723G7, Tosoh, Shanghai, China). Plasma concentrations of NT-proBNP were measured by the electrochemiluminescence immunoassay "Roche Diagnostics Elecsys 2010 system" (USA), and the reference range was $0 \mathrm{pg} / \mathrm{ml}$ to $600 \mathrm{pg} / \mathrm{ml}$.

\section{MetS definition}

We used the definition of MetS recommended by the Chinese Diabetes Society of the Chinese Medical Association for the Chinese population. Thus, individuals were defined as having MetS if they satisfied $\geq 3$ of the following 4 criteria: (1) overweight or obese or elevated body mass index $\left(\geq 25.0 \mathrm{~kg} / \mathrm{m}^{2}\right),(2)$ high fasting plasma glucose ( $\geq 6.1 \mathrm{mmol} / \mathrm{l})$ and/or postprandial blood glucose $(\geq 7.8 \mathrm{mmol} / \mathrm{l})$ and/or use of hypoglycaemic medications, (3) high blood pressure systolic/ diastolic $\geq 140 / 90 \mathrm{mmHg}$ or use of antihypertensive medications, (4) high fasting triglycerides $(\geq 1.7 \mathrm{mmol} / \mathrm{l})$ and/or reduced high density lipoprotein cholesterol (<0.9 mmol// in men, $<1.0 \mathrm{mmol} / \mathrm{l}$ in women).

\section{Echocardiography}

Echocardiography examinations were performed with a Vingmed System 5 Doppler echocardiographic unit (GE Vingmed Ultrasound, Horten, Norway). Conventional echocardiography measurements were performed according to American Society of Echocardiography guidelines. Left ventricular mass (LVM) was calculated using the Devereux formula as follows: $\operatorname{LVM}(\mathrm{g})=1.04 \times$ $\left[(\text { LVIDd + LVPWd + IVSd })^{3}-\right.$ LVIDd $\left.^{3}\right]-13.6$, where LVIDd represents left ventricular diameter in end diastole $(\mathrm{cm})$, LVPWd represents left ventricular posterior wall thickness in diastole $(\mathrm{cm})$, and IVSd represents interventricular septal thickness in end diastole $(\mathrm{cm})$. Left ventricular mass was corrected for body surface area (BSA) to give LVM index (LVMI). Left atrial diameter (LAD) and aortic root dimension (AOD) were also measured. Left ventricular systolic function was assessed by 
calculation of left ventricular ejection fraction (LVEF). Diastolic function was assessed by determining the E-to-A ratio (E/A), where $E$ and $A$ represent the early and late velocities respectively.

\section{Statistical analysis}

We used natural logarithmic (Log) transformations of peptide levels because NT-proBNP followed approximately lognormal distributions. Furthermore, the association between the elevation of NT-proBNP and potential metabolic factors was investigated by multiple linear regression analysis. Multiple linear regression analysis was performed to relate Log NT-proBNP to metabolic variables that included gender, body mass index, high density lipoprotein cholesterol, low density lipoprotein cholesterol, systolic blood pressure, diastolic blood pressure, fasting plasma glucose, and postprandial blood glucose. This model also included age, serum creatinine, AOD, LAD, LVMI, LVEF and E/A.

Next, considering differences of NT-proBNP levels between MetS and non-MetS groups with disturbance of the possible confounding variables, we performed covariance analysis with adjustment for age, gender, serum creatinine, LAD, AOD, LVMI and LVEF which have been identified by multiple regression analysis.

In order to better investigate the association of NT-proBNP and the components of MetS, we compared NT-proBNP levels of different subgroups by covariance analysis.

Results were analysed using the Statistical Package for Social Sciences for Windows ver. 13.0 (SPSS, Chicago, IL, USA). Continuous variables were expressed as mean \pm SD or median (range) values. Tests were two-sided and a $p$-value of $<0.05$ was considered significant.

\section{Results}

Characteristics of the study sample $(n=469)$ are shown in Table I. There are 124 males and 106 females (mean age, $65.2 \pm 12.0$ years) in the MetS group and 142 males and 97 females (mean age, $66.0 \pm 12.6$ years) in the non-MetS group. As expected, body mass index, systolic blood pressure, diastolic blood pressure, fasting blood glucose, postprandial blood glucose, and lipid and lipoprotein profile were significantly different between the MetS group and the non-MetS group ( $p<0.001)$. Hypertension and diabetes were more prevalent in the patients with MetS $(p<0.001)$.

\section{NT-proBNP and metabolic factors}

Results of multiple linear regression models relating Log NT-proBNP with metabolic risk factors are shown in Table II. In all cases, a negative association was observed between plasma
NT-proBNP levels and body mass index $(p=0.016)$, as previously described. Additionally, NT-proBNP levels were inversely associated with diastolic blood pressure $(p=0.015)$, fasting blood glucose $(p=0.046)$, triglycerides $(p=0.009)$, low density lipoprotein cholesterol $(p=0.036)$, and LVEF $(p \leq 0.001)$. NT-proBNP was positively associated with age $(p<0.001)$, systolic blood pressure $(p=0.004)$, high density lipoprotein cholesterol $(p=0.013)$ and LVMI $(p<0.001)$. Plasma NT-proBNP was negatively associated with systolic blood pressure $(p=0.001)$ and fasting blood glucose $(p=0.015)$ in women but not in men. However, the association for triglycerides $(p=0.002)$ and high density lipoprotein cholesterol $(p=0.014)$ was significant in men but not in women.

\section{Log NT-proBNP levels in patients with MetS and without MetS}

The differences between the two groups of patients in terms of their NT-proBNP levels are displayed in Figure 1 and Table III. Plasma levels of log NT-proBNP are lower in all participants with MetS after adjustment for age, gender (for total patients), serum creatinine, LAD, AOD, LVMI, and LVEF ( $p=0.001)$. Similarly, the decrease of NT-proBNP levels in patients with MetS remained significant for men $(p=0.038)$ and for women ( $p=0.022)$ after the same adjustment for age, and clinical and echocardiographic characteristics.

\section{Log NT-proBNP levels in patients of MetS subgroups and non-MetS}

Next, we divided the subjects with MetS into subgroups according to their body mass index, systolic blood pressure, diastolic blood pressure, fasting blood glucose, triglycerides or high density lipoprotein cholesterol. In men, the cut points are $25.0 \mathrm{~kg} / \mathrm{m}^{2}, 140 \mathrm{mmHg}, 90 \mathrm{mmHg}, 6.1 \mathrm{mmol} / \mathrm{l}$, $1.7 \mathrm{mmol} / /$ or $0.9 \mathrm{mmol} / /$ respectively. In women, the cut points are similar to those of men except that high density lipoprotein cholesterol is $1.0 \mathrm{mmol} / \mathrm{l}$. Covariance analyses were conducted and adjusted for age, gender (for total patients), serum creatinine, LAD, AOD, LVMI, and LVEF. In addition, for example, accounting for the other MetS components, such as dyslipidaemia, hyperglycaemia and elevated blood pressure, may influence comparison of log NT-proBNP levels between MetS subgroups stratified by BMI and the non-MetS group. So here the analyses of covariance were also adjusted for the other MetS components except for the categorical exposure variable (like BMI in the above-mentioned example). The MetS components above referred to body mass index, triglycerides, high density lipoprotein cholesterol, low density lipoprotein cholesterol, fasting blood glucose, 
Yuanyuan Bao, Xiliang Shang, Linuo Zhou, Renming Hu, Yiming Li, Wei Ding

Table I. Clinical characteristics of the study participants with and without MetS

\begin{tabular}{|c|c|c|c|}
\hline Parameters & MetS(+) $n=230$ & MetS(-) $n=239$ & Value of $p$ \\
\hline Sex, no., male/female & $124 / 106$ & $142 / 97$ & \\
\hline Age [year] & $65.2 \pm 12.0$ & $66.0 \pm 12.6$ & 0.689 \\
\hline Smoking [\%] & 42 & 43 & 0.624 \\
\hline Hypertension [\%] & 53 & 11 & $<0.001$ \\
\hline Diabetes mellitus [\%] & 72 & 30 & $<0.001$ \\
\hline Body mass index $\left[\mathrm{kg} / \mathrm{m}^{2}\right]$ & $27.0 \pm 4.5$ & $23.3 \pm 4.2$ & $<0.001$ \\
\hline $\mathrm{FBG}[\mathrm{mmol} / \mathrm{l}]$ & $6.6 \pm 1.2$ & $5.2 \pm 1.0$ & $<0.001$ \\
\hline $\mathrm{PBG}[\mathrm{mmol} / \mathrm{l}]$ & $9.3 \pm 3.0$ & $7.3 \pm 2.8$ & $<0.001$ \\
\hline Glycated haemoglobin [\%] & $6.8 \pm 0.8$ & $5.8 \pm 0.7$ & $<0.001$ \\
\hline Systolic blood pressure [mmHg] & $144(85)$ & $130(75)$ & $<0.001$ \\
\hline Diastolic blood pressure [mmHg] & $89(45)$ & $77(35)$ & $<0.001$ \\
\hline LDL cholesterol [mmol/l] & $2.61 \pm 0.8$ & $2.59 \pm 0.8$ & 0.301 \\
\hline HDL cholesterol [mmol/l] & $0.9 \pm 0.2$ & $1.2 \pm 0.3$ & $<0.001$ \\
\hline Triglycerides [mmol/l] & $2.2 \pm 1.3$ & $1.3 \pm 0.6$ & $<0.001$ \\
\hline Serum creatinine $[\mu \mathrm{mol} / \mathrm{l}]$ & $79.5 \pm 25.1$ & $79.3 \pm 22.7$ & 0.175 \\
\hline $\mathrm{LAD}[\mathrm{mm}]$ & $40.0 \pm 7.1$ & $38.2 \pm 5.9$ & 0.238 \\
\hline $\mathrm{AOD}[\mathrm{mm}]$ & $33.0 \pm 3.4$ & $33.0 \pm 4.5$ & 0.642 \\
\hline LVM [g] & $228.2 \pm 64.1$ & $195.5 \pm 61.5$ & $<0.001$ \\
\hline LVMI $\left[\mathrm{g} / \mathrm{m}^{2}\right]$ & $127.3 \pm 31.9$ & $119.1 \pm 40.5$ & 0.023 \\
\hline $\mathrm{EF}[\%]$ & $62.7 \pm 11.7$ & $64.5 \pm 9.5$ & 0.091 \\
\hline E/A ratio & $1.3 \pm 0.2$ & $1.4 \pm 0.2$ & 0.689 \\
\hline \multicolumn{4}{|l|}{ Medication [\%] } \\
\hline Calcium antagonists & 25.3 & 23.2 & 0.331 \\
\hline$\beta$-blockers & 50.8 & 49.6 & 0.782 \\
\hline ACE inhibitors & 35.4 & 33.1 & 0.534 \\
\hline
\end{tabular}

FBG - fasting blood glucose, PBG - postprandial blood glucose, HDL cholesterol - high density lipoprotein cholesterol, LDL cholesterol - low density lipoprotein cholesterol, $L A D$ - left atrial diameter, $A O D$ - aortic root dimension, $E F$ - ejection fraction, $E$ - transmitral early diastolic velocity, A - transmitral late diastolic velocity, E/A - ratio of transmitral early (E) and late (A) diastolic velocities, IVS - interventricular septum, $P W$ - posterior wall, LVM - left ventricular mass, LVMI - left ventricular mass index, values are mean \pm SD when appropriate

Table II. Multivariable association of metabolic risk factors with log NT-proBNP levels

\begin{tabular}{|c|c|c|c|c|c|c|}
\hline \multirow[t]{2}{*}{ Independent variables } & \multicolumn{2}{|c|}{ Total } & \multicolumn{2}{|c|}{ Men } & \multicolumn{2}{|c|}{ Women } \\
\hline & $\beta$ & $p$ & $\beta$ & $p$ & $\beta$ & $p$ \\
\hline Age & 0.337 & $<0.001$ & 0.342 & $<0.001$ & 0.387 & $<0.001$ \\
\hline Gender & 0.221 & 0.004 & & & & \\
\hline Body mass index & -0.173 & 0.007 & -0.252 & 0.003 & -0.223 & 0.018 \\
\hline Systolic blood pressure & 0.217 & 0.004 & 0.114 & 0.225 & 0.372 & 0.001 \\
\hline Diastolic blood pressure & -0.179 & 0.015 & -0.186 & 0.034 & -0.397 & 0.007 \\
\hline Fasting blood glucose & -0.150 & 0.046 & -0.176 & 0.093 & -0.276 & 0.015 \\
\hline Triglycerides & -0.265 & 0.009 & -0.351 & 0.002 & 0.204 & 0.285 \\
\hline HDL cholesterol & 0.240 & 0.013 & 0.317 & 0.014 & 0.014 & 0.922 \\
\hline LDL cholesterol & -0.423 & 0.036 & -0.456 & 0.170 & 0.102 & 0.715 \\
\hline LVMI & 0.315 & $<0.001$ & 0.292 & 0.001 & 0.452 & $<0.001$ \\
\hline LVEF & -0.377 & $<0.001$ & -0.446 & $<0.001$ & -0.372 & $<0.001$ \\
\hline
\end{tabular}

FBG - fasting blood glucose, HDL cholesterol - high density lipoprotein cholesterol, LDL cholesterol - low density lipoprotein cholesterol, LVMI - left ventricular mass index, LVEF - left ventricular ejection fraction 
systolic blood pressure and diastolic blood pressure when internalized as covariates.

The differences between patients of MetS subgroups and non-MetS in terms of their NT-proBNP levels are displayed in Figure 2 and Tables IV-VIII. For all subjects totally, Log NT-proBNP concentrations were lower in MetS subjects with dyslipidaemia, hyperglycaemia, elevated body mass index and diastolic blood pressure than those without MetS ( $p<0.05$ ), but did not reach significance in MetS subjects with normal blood fat, glucose, body mass index, systolic blood pressure and diastolic blood pressure compared to those without MetS ( $p=\mathrm{NS}$ ). However, Log NT-proBNP levels were higher in MetS patients with elevated systolic blood pressure than non-MetS patients $(p<0.05)$. Similar findings were obtained in men and women except for women with elevated body mass index.

\section{Discussion}

$\mathrm{N}$-terminal pro-B-type natriuretic peptide (NT-proBNP), the inactive amino terminal fragment, and the biologically active form of B-type natriuretic peptide (BNP), are cleavage products of the precursor pre-proBNP which is synthesized by ventricular myocytes in response to physiological signals such as stretching of the ventricular wall or changes in systemic blood pressure, sodium levels or extracellular volume. B-type natriuretic peptide is metabolized by specific natriuretic peptide receptors found in the kidney, lung, liver, and along the vascular endothelium, while NT-proBNP is mainly cleared by the kidneys. It has recently been recognized as a new and useful biochemical marker for both diagnosis and ruling out of congestive heart failure (CHF) [1]. For the present, assessment of plasma NT-proBNP concentration is of potential value for risk of death and cardiovascular complications in the general population and may be useful for detection of a symptomatic left ventricular systolic dysfunction and systolic dysfunction in the general population [7, 8]. Recent clinical trials have also shown that NT-proBNP is elevated in patients with acute coronary syndrome (ACS) and exercise training in patients after myocardial infarction could decrease plasma NT-proBNP levels [9, 10]. Baghdady et al. observed an increase of NT-proBNP levels in ventricular septal defect with cardiac decompensation, and it is correlated with clinical score and echocardiography parameters [11].

\section{NT-proBNP and metabolic syndrome}

An inverse relationship between BNP and MetS has been described previously in the Framingham Heart Study [2]. In addition, levels of NT-proBNP

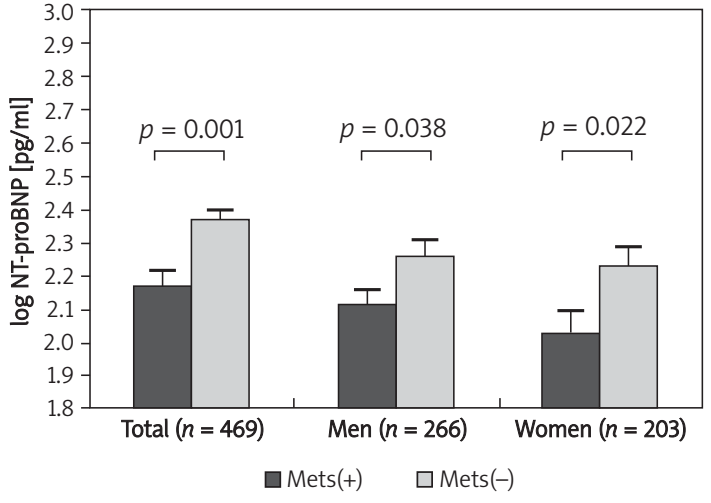

Figure 1. Adjusted log NT-proBNP levels in patients with Mets and without Mets

MetS - metabolic syndrome, NT-proBNP - N-terminal pro-Btype natriuretic peptide. Covariance analysis between groups was carried out. Levels were obtained by log transformation of NT-proBNP levels adjusted for age, gender, serum creatinine, LAD, AOD, LVMI, and LVEF. Data are presented as mean $\pm S D$

have also been detected to be significantly lower in MetS [6]. Consistent with the above-mentioned finding, differences of NT-proBNP plasma concentrations were established between MetS and nonMetS in our study by covariance analysis after adjustment for age, gender (for total patients), serum creatinine, LAD, AOD, LVMI, and LVEF. On the other hand, to the best of our knowledge, ours is the first investigation to demonstrate that plasma NT-proBNP is lower in patients with MetS defined by the Chinese Diabetes Society of the Chinese Medical Association.

\section{NT-proBNP, body mass index and blood lipid}

Consistent with prior experimental observations [12], we found that there is a strong relationship between serum NT-proBNP and plasma triglycerides, high density lipoprotein cholesterol, and low density lipoprotein cholesterol levels, suggesting a strong relationship between NT-proBNP and lipid metabolism. An inverse relationship between serum NT-proBNP and cholesterol has been described previously and has been hypothesized to be a potential link impairing the natural blood pressure regulation [6]. Our data support to some degree this hypothesis because of the inverse relationship between low density lipoprotein cholesterol and NT-proBNP. Log NT-proBNP levels were also found to be significantly lower in patients of MetS subgroups stratified by plasma triglycerides and high density lipoprotein cholesterol compared to those without MetS, suggesting a negative association between lower NT-proBNP concentration and hyperlipidaemia.

We also found that there is a strong relationship between serum NT-proBNP and body mass index. An inverse relationship between serum BNP and body mass index has been described recently [13], 
A

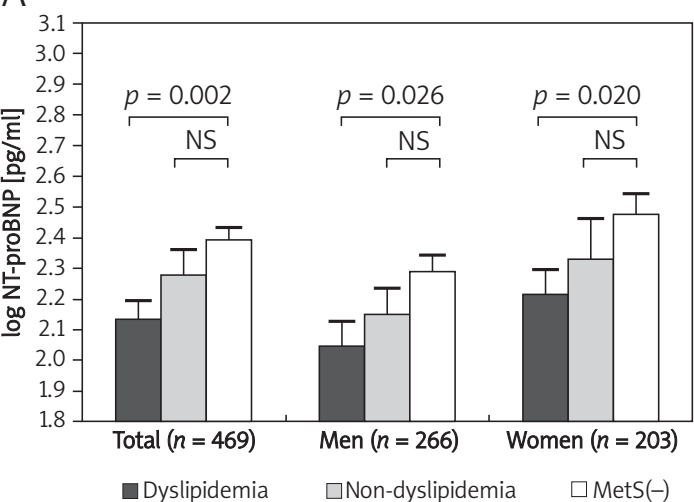

C

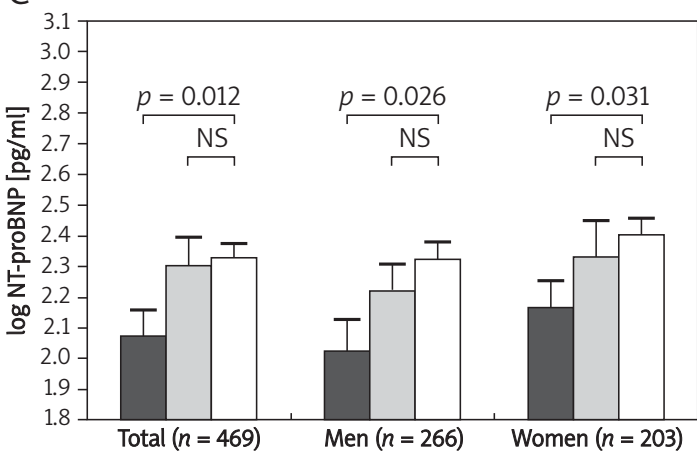

$\square \mathrm{FPG} \geq 6.1 \mathrm{mmol} / \mathrm{l} \quad \square \mathrm{FPG}<6.1 \mathrm{mmol} / \mathrm{l} \quad \square \mathrm{MetS}(-)$
B

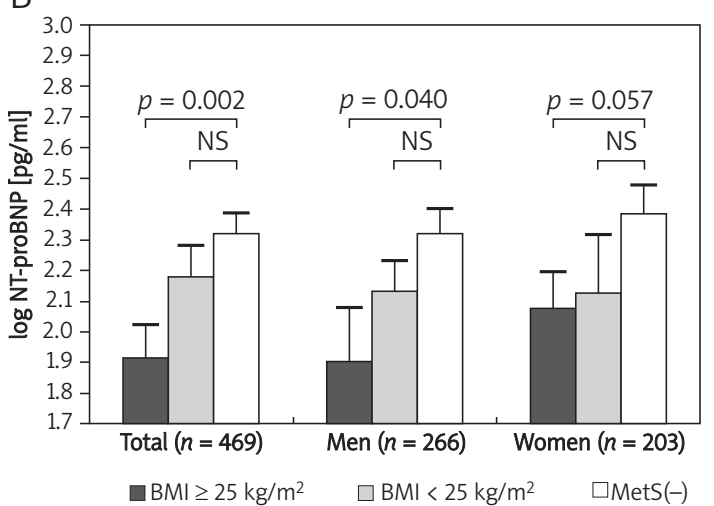

D

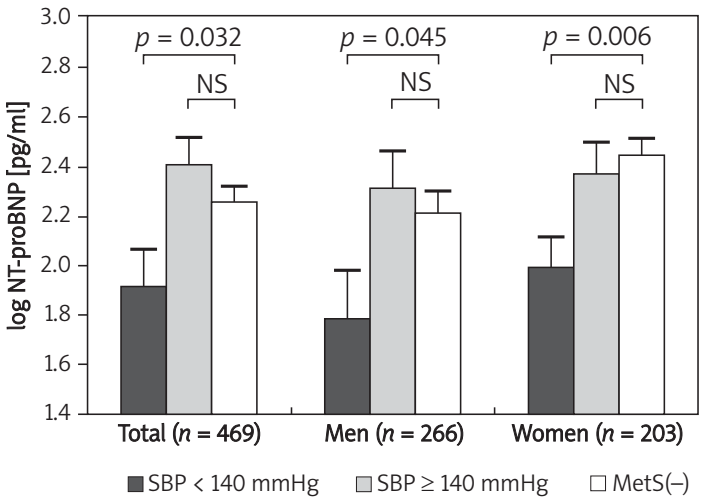

$\mathrm{E}$

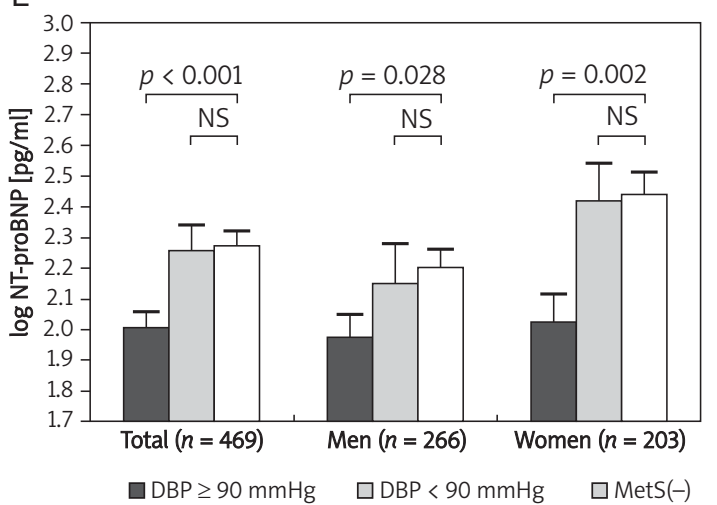

Figure 2. Adjusted log NT-proBNP levels in subgroups of MetS and patients without MetS

MetS - metabolic syndrome, NT-proBNP - N-terminal pro-B-type natriuretic peptide. Levels were obtained by log transformation of NT-proBNP levels. Covariance analysis between groups was carried out and adjusted for age, serum creatinine, left atrial diameter, left ventricular mass, left ventricular ejection fraction and the other MetS covariates. Datas are presented as mean \pm SD; NS - no significant

Table III. Adjusted log NT-proBNP levels in patients with MetS and without MetS

\begin{tabular}{|lcccccc|}
\hline & \multicolumn{2}{c}{ Total } & \multicolumn{2}{c|}{ Men } & \multicolumn{2}{c|}{ Women } \\
\cline { 2 - 7 } & $n$ & Log NT-proBNP & $n$ & Log NT-proBNP & $n$ & Log NT-proBNP \\
\hline MetS(+) & 230 & $2.173 \pm 0.044$ & 124 & $2.110 \pm 0.056$ & 106 & $2.022 \pm 0.074$ \\
\hline MetS(-) & 239 & $2.367 \pm 0.035$ & 142 & $2.261 \pm 0.045$ & 97 & $2.232 \pm 0.055$ \\
\hline Value of $p$ & 0.001 & 0.038 & & 0.022 \\
\hline
\end{tabular}

MetS - metabolic syndrome, NT-proBNP - N-terminal pro-B-type natriuretic peptide. Covariance analysis between groups was carried out. Levels were obtained by log transformation of NT-proBNP levels adjusted for age, gender, serum creatinine, LAD, AOD, LVMI, and LVEF. Data are presented as mean $\pm S D$ 
Table IV. Adjusted log NT-proBNP levels in blood lipid subgroups of MetS and patients without MetS

\begin{tabular}{|lcccccc|}
\hline & \multicolumn{2}{c}{ Total } & \multicolumn{2}{c}{ Men } & \multicolumn{2}{c|}{ Women } \\
\cline { 2 - 7 } & $n$ & Log NT-proBNP & $n$ & Log NT-proBNP & $n$ & Log NT-proBNP \\
\hline MetS(-) & 239 & $2.391 \pm 0.044$ & 142 & $2.289 \pm 0.056$ & 97 & $2.476 \pm 0.063$ \\
\hline MetS(+) & 230 & & 124 & & 106 & \\
\hline Non-dyslipidaemia & 110 & $\begin{array}{c}2.275 \pm 0.084 \\
(p=0.243)\end{array}$ & 51 & $\begin{array}{c}2.145 \pm 0.093 \\
(p=0.213)\end{array}$ & 59 & $\begin{array}{c}2.329 \pm 0.135 \\
(p=0.342)\end{array}$ \\
\hline Dyslipidaemia & 120 & $\begin{array}{c}2.133 \pm 0.059 \\
(p=0.002)\end{array}$ & 73 & $\begin{array}{c}2.044 \pm 0.082 \\
(p=0.026)\end{array}$ & 47 & $\begin{array}{c}2.213 \pm 0.084 \\
(p=0.020)\end{array}$ \\
\hline
\end{tabular}

MetS - metabolic syndrome, NT-proBNP - N-terminal pro-B-type natriuretic peptide. Covariance analysis between groups was carried out. Levels were obtained by log transformation of NT-proBNP levels adjusted for age, gender, serum creatinine, LAD, AOD, LVMI, and LVEF. Data are presented as mean $\pm S D$

Table V. Adjusted log NT-proBNP levels in BMI subgroups of MetS and patients without MetS

\begin{tabular}{|lcccccc|}
\hline & \multicolumn{2}{c}{ Total } & \multicolumn{2}{c}{ Men } & \multicolumn{2}{c|}{ Women } \\
\cline { 2 - 7 } & $n$ & Log NT-proBNP & $n$ & Log NT-proBNP & $n$ & Log NT-proBNP \\
\hline MetS(-) & 239 & $2.317 \pm 0.068$ & 142 & $2.317 \pm 0.087$ & 97 & $2.388 \pm 0.090$ \\
\hline MetS(+) & 230 & & 124 & & 106 & \\
\hline BMI $<25 \mathrm{~kg} / \mathrm{m}^{2}$ & 89 & $\begin{array}{c}2.182 \pm 0.099 \\
(p=0.257)\end{array}$ & 46 & $\begin{array}{c}2.130 \pm 0.100 \\
(p=0.147)\end{array}$ & 43 & $\begin{array}{c}2.126 \pm 0.190 \\
(p=0.214)\end{array}$ \\
\hline BMI $\geq 25 \mathrm{~kg} / \mathrm{m}^{2}$ & 141 & $\begin{array}{c}1.916 \pm 0.108 \\
(p=0.002)\end{array}$ & 78 & $\begin{array}{c}1.904 \pm 0.175 \\
(p=0.040)\end{array}$ & 63 & $\begin{array}{c}2.074 \pm 0.127 \\
(p=0.057)\end{array}$ \\
\hline
\end{tabular}

MetS - metabolic syndrome, NT-proBNP - N-terminal pro-B-type natriuretic peptide. Covariance analysis between groups was carried out. Levels were obtained by log transformation of NT-proBNP levels adjusted for age, gender, serum creatinine, LAD, AOD, LVMI, and LVEF. Data are presented as mean $\pm S D$

Table VI. Adjusted log NT-proBNP levels in FPG subgroups of MetS and patients without MetS

\begin{tabular}{|lcccccc|}
\hline & \multicolumn{2}{c}{ Total } & \multicolumn{2}{c}{ Men } & \multicolumn{2}{c|}{ Women } \\
\cline { 2 - 7 } & $n$ & Log NT-proBNP & $n$ & Log NT-proBNP & $n$ & Log NT-proBNP \\
\hline MetS(-) & 239 & $2.325 \pm 0.046$ & 142 & $2.323 \pm 0.055$ & 97 & $2.399 \pm 0.054$ \\
\hline MetS(+) & 230 & & 124 & & 106 & \\
\hline FPG $<6.1 \mathrm{mmol} / \mathrm{l}$ & 105 & $\begin{array}{c}2.296 \pm 0.100 \\
(p=0.795)\end{array}$ & 55 & $\begin{array}{c}2.218 \pm 0.088 \\
(p=0.328)\end{array}$ & 50 & $\begin{array}{c}2.328 \pm 0.119 \\
(p=0.602)\end{array}$ \\
\hline FPG $\geq 6.1 \mathrm{mmol} / \mathrm{l}$ & 125 & $\begin{array}{c}2.068 \pm 0.089 \\
(p=0.012)\end{array}$ & 69 & $\begin{array}{c}2.021 \pm 0.104 \\
(p=0.026)\end{array}$ & 56 & $\begin{array}{c}2.165 \pm 0.085 \\
(p=0.031)\end{array}$ \\
\hline
\end{tabular}

MetS - metabolic syndrome, NT-proBNP - N-terminal pro-B-type natriuretic peptide. Covariance analysis between groups was carried out. Levels were obtained by log transformation of NT-proBNP levels adjusted for age, gender, serum creatinine, LAD, AOD, LVMI, and LVEF. Data are presented as mean $\pm S D$

Table VII. Adjusted log NT-proBNP levels in SBP subgroups of MetS and patients without MetS

\begin{tabular}{|lcccccc|}
\hline & \multicolumn{2}{c}{ Total } & \multicolumn{2}{c|}{ Men } & \multicolumn{2}{c|}{ Women } \\
\cline { 2 - 7 } & $n$ & Log NT-proBNP & $n$ & Log NT-proBNP & $n$ & Log NT-proBNP \\
\hline MetS(-) & 239 & $2.258 \pm 0.071$ & 142 & $2.214 \pm 0.088$ & 97 & $2.445 \pm 0.077$ \\
\hline MetS(+) & 230 & & 124 & & 106 & \\
\hline SBP $<140 \mathrm{mmHg}$ & 109 & $\begin{array}{c}1.919 \pm 0.147 \\
(p=0.032)\end{array}$ & 56 & $\begin{array}{c}1.795 \pm 0.188 \\
(p=0.045)\end{array}$ & 53 & $\begin{array}{c}1.991 \pm 0.124 \\
(p=0.006)\end{array}$ \\
\hline SBP $\geq 140 \mathrm{mmHg}$ & 121 & $\begin{array}{c}2.416 \pm 0.106 \\
(p=0.212)\end{array}$ & 68 & $\begin{array}{c}2.316 \pm 0.150 \\
(p=0.560)\end{array}$ & 53 & $\begin{array}{c}2.375 \pm 0.120 \\
(p=0.643)\end{array}$ \\
\hline
\end{tabular}

MetS - metabolic syndrome, NT-proBNP - N-terminal pro-B-type natriuretic peptide. Covariance analysis between groups was carried out. Levels were obtained by log transformation of NT-proBNP levels adjusted for age, gender, serum creatinine, LAD, AOD, LVMI, and LVEF. Data are presented as mean $\pm S D$ 
Table VIII. Adjusted log NT-proBNP levels in DBP subgroups of MetS and patients without MetS

\begin{tabular}{|c|c|c|c|c|c|c|}
\hline & \multicolumn{2}{|c|}{ Total } & \multicolumn{2}{|c|}{ Men } & \multicolumn{2}{|c|}{ Women } \\
\hline & $n$ & Log NT-proBNP & $n$ & Log NT-proBNP & $n$ & Log NT-proBNP \\
\hline MetS(-) & 239 & $2.273 \pm 0.045$ & 142 & $2.200 \pm 0.058$ & 97 & $2.439 \pm 0.073$ \\
\hline MetS(+) & 230 & & 124 & & 106 & \\
\hline $\mathrm{DBP}<90 \mathrm{mmHg}$ & 107 & $\begin{array}{c}2.250 \pm 0.091 \\
(p=0.824)\end{array}$ & 59 & $\begin{array}{c}2.146 \pm 0.132 \\
(p=0.715)\end{array}$ & 48 & $\begin{array}{c}2.420 \pm 0.123 \\
(p=0.906)\end{array}$ \\
\hline $\mathrm{DBP} \geq 90 \mathrm{mmHg}$ & 123 & $\begin{array}{c}2.001 \pm 0.059 \\
(p<0.001)\end{array}$ & 65 & $\begin{array}{c}1.974 \pm 0.078 \\
(p=0.028)\end{array}$ & 58 & $\begin{array}{c}2.024 \pm 0.092 \\
(p=0.002)\end{array}$ \\
\hline
\end{tabular}

MetS - metabolic syndrome, NT-proBNP - N-terminal pro-B-type natriuretic peptide. Covariance analysis between groups was carried out. Levels were obtained by log transformation of NT-proBNP levels adjusted for age, gender, serum creatinine, LAD, AOD, LVMI, and LVEF. Data are presented as mean $\pm S D$

which is also supported to some degree by our data. This is also supported by previous observations that obese subjects are more sensitive to sodium load on account of a reduced effect of atrial natriuretic peptide, partly attributable to increased removal of natriuretic peptide from the circulation by adipocytes through abundant natriuretic peptide clearance receptors in adipose tissue of obese people [14]. NT-proBNP may also relate to this, but the importance of the natriuretic clearance receptor for clearance of NT-proBNP is unknown [15]. Alternatively, NT-proBNP may, through lipolytic and lipomobilizing effects, change the metabolic state as demonstrated for atrial natriuretic peptide [16] and reduce the incidence of overweight and obesity. An association between natriuretic peptides and levels of the insulin-sensitizing hormone adiponectin has been reported, with low levels of BNP associated with low levels of adiponectin despite adjustment for body mass index [17] Natriuretic peptides also stimulate lipolysis and release of triacylglycerols from adipose tissue [18]. Reduced natriuretic peptide signalling could have detrimental effects via the promotion of lipid accumulation in adipose tissue and skeletal muscle.

\section{NT-proBNP and blood glucose}

An inverse relationship between plasma NT-proBNP levels and fasting blood glucose was found in our study. Log NT-proBNP levels were also found to be significantly lower in patients of MetS subgroups stratified by fasting blood glucose compared to non-MetS subjects. Some experimental observations suggest that low natriuretic peptide levels could predispose to insulin resistance. Reduced natriuretic peptide activity leads to greater activation of the renin-angiotensin system [19]. Activation of the renin-angiotensin system promotes the development of insulin resistance via multiple mechanisms [20], including inhibition of intracellular insulin signalling, enhanced oxidative stress, inflammation, reduced adipocyte differentiation, and decreased perfusion to the skeletal muscle and pancreas.

\section{Natriuretic peptides and blood pressure}

Previous studies have reported a positive association between plasma natriuretic peptide levels and systolic blood pressure [21]. In conformity with these data, we also found that low NT-proBNP levels were associated with almost all component of the metabolic syndrome except elevated systolic blood pressure, which was associated with higher plasma NT-proBNP levels, indicating the haemodynamic influence of blood pressure on natriuretic peptide synthesis. The observation that plasma NT-proBNP levels relate differently to systolic blood pressure than to other metabolic components suggests that blood pressure segregates separately from other MetS components [22]. In the Insulin Resistance Atherosclerosis Study, Hanley et al. [22] used principal factor analysis to identify 2 "factors". One is a metabolic factor, consisting of body mass index, glycaemic measures, triglycerides, and high density lipoprotein cholesterol; the other is a blood pressure factor, comprising systolic and diastolic blood pressure. HOMA-IR and fasting insulin correlated with the metabolic factor but not with the blood pressure factor. These data suggest that blood pressure may have a different pathophysiological mechanism from other metabolic components, which may help to interpret the different association between systolic blood pressure and NT-proBNP from others. There is an inverse correlation between plasma NT-proBNP levels and diastolic blood pressure, which may reflect the influence of pulse pressure [6]. Increased pulse pressure is often accompanied by lower diastolic blood pressure, particularly in older individuals.

\section{NT-proBNP and other risk factors}

The association between left ventricular hypertrophy and plasma levels of NT-proBNP is consistent. Some studies have found an association between NT-proBNP levels and left ventricular hypertrophy, whereas others have not [8]. In the present study we found there was an independent 
association between left ventricular mass index and NT-proBNP levels in subjects.

Age has been shown to influence circulating natriuretic peptide levels, and the magnitude of the effects and potential importance in the interpretation of NT-proBNP remain unclear [23]. A possible explanation for increased NT-proBNP levels with age may be increased age-related fibrosis, diastolic dysfunction and reduced renal clearance [24]. In this study we also found that NT-proBNP levels were positively related to age in all subjects.

\section{Comparison with previous studies}

An inverse association between plasma natriuretic peptide levels and obesity has previously been described in the Framingham [2] and other studies [13, 25]. However, few studies have investigated the relations between NT-proBNP levels and metabolic components, especially in MetS subjects diagnosed according to recommendations of the Chinese Diabetes Society. Inconsistent associations have been reported between plasma natriuretic peptides and glycaemic status [5] or plasma lipids [4]. In the population-based Danish cohort of Olsen et al. [6], plasma NT-proBNP levels were inversely associated with serum total cholesterol, serum triglycerides and plasma glucose. The present study differs from those findings by the use of covariance analyses and the ability to adjust for differences in age, gender, renal function with serum creatinine, LAD, AOD, LVMI, and LVEF. In addition, as gender is an independent factor influencing NT-proBNP levels, we divided all subjects into male and female groups. For men, fasting blood glucose was not an independent predictor of NT-proBNP, while for women NTproBNP levels were not independently correlated with blood lipid. Notwithstanding the above, the similarity in study findings provides convincing evidence of an association between the natriuretic peptide and metabolic components.

Several limitations of the study deserve comment. First, the study results indicated that NT-proBNP is different in MetS subjects from nonMetS. The fact that NT-proBNP was related to metabolic components independently of age and clinical and echocardiographic characteristics indicates that NT-proBNP is a superior marker of metabolic syndrome and therefore suitable to detect subclinical MetS before it develops into clinical disease. However, the positive or negative relationship between NT-proBNP and metabolic components may be a problem when defining cut-off values for varying levels of obesity, dyslipidaemia, or hyperglycaemia. Here subgroups are established according to the Chinese criteria. Next, further studies are required to investigate
NT-proBNP levels in MetS subgroups by other traditional cut-off levels in patients. Second, the multiple regression models explained only a moderate influence of potential risk factors on NT-proBNP levels. Other environmental factors may contribute to the unexplained variation. Finally, it is important to mention that our study concerned Chinese individuals and our findings may not be relevant to those of other countries.

The diagnostic value of plasma natriuretic peptide measurements has been demonstrated in a variety of settings. The range of factors that may influence the biomarker needs to be understood for interpretation of the results. Previous studies have shown the use of lower natriuretic peptide cut points for diagnosing heart failure in obese individuals [25]. Our data raise the possibility that other metabolic components should be taken into account when plasma natriuretic peptide (NT-proBNP) measurements are interpreted. But whether diagnostic thresholds should be altered in individuals with the metabolic syndrome is unknown and needs further investigation in prospective studies. In addition to that, because natriuretic peptides play an important role in the counter-regulatory response to volume and pressure overload, it is possible that lower natriuretic peptide levels contribute to the susceptibility of individuals with the metabolic syndrome to hypertension and left ventricular hypertrophy.

In conclusion, plasma NT-proBNP levels were lower in patients with MetS, especially in MetS patients with dyslipidaemia, elevated body mass index, fasting blood glucose and diastolic blood pressure. Our data raise the possibility that reduced plasma NT-proBNP levels may be a marker for MetS, which might possess significant clinical and pathophysiological implications. More prospective studies are needed regarding the relations of lower NT-proBNP levels and sodium, insulin resistance, and the impact of reduced natriuretic peptide levels on cardiovascular events in MetS.

\section{References}

1. Wu CJ, Chang HW, Hung WC, et al. N-terminal pro-brain natriuretic peptide is a biomarker of congestive heart failure and predictive of 30-day untoward clinical outcomes in patients with acute myocardial infarction undergoing primary percutaneous coronary intervention. Circ J 2006; 70: 163-8.

2. Wang TJ, Larson MG, Levy D, et al. The impact of obesity on plasma natriuretic peptide levels: the Framingham Heart Study. Circulation 2004; 109: 594-600.

3. Das SR, Drazner MH, Dries DL, et al. Impact of body mass and body composition on circulating levels of natriuretic peptides: results from the Dallas Heart Study. Circulation 2005; 112: 2163-8.

4. Kanda $\mathrm{H}$, Kita $\mathrm{Y}$, Okamura $\mathrm{T}$, et al. What factors are associated with high plasma B-type natriuretic peptide 
levels in a general Japanese population? J Hum Hypertens 2005; 19: 165-72

5. Yano Y, Katsuki A, Gabazza EC, et al. Plasma brain natriuretic peptide levels in normotensive noninsulindependent diabetic patients with microalbuminuria. J Clin Endocrinol Metab 1999; 84: 2353-6.

6. Olsen MH, Hansen TW, Christensen MK, et al. N-terminal pro brain natriuretic peptide is inversely related to metabolic cardiovascular risk factors and the metabolic syndrome. Hypertension 2005; 46: 660-6.

7. Ceyhan C, Unal S, Yenisey C, Tekten T, Ceyhan FB. The role of $\mathrm{N}$ terminal pro-brain natriuretic peptide in the evaluation of left ventricular diastolic dysfunction: correlation with echocardiographic indexes in hypertensive patients. Int J Cardiovasc Imaging 2008; 24: 253-9.

8. Talwar S, Siebenhofer A, Williams B, Ng L. Influence of hypertension, left ventricular hypertrophy, and left ventricular systolic dysfunction on plasma $\mathrm{N}$ terminal proBNP. Heart 2000; 83: 278-82.

9. Ogawa A, Seino Y, Yamashita T, Ogata k, Takano T. Difference in elevation of $\mathrm{N}$-terminal pro-BNP and conventional cardiac markers between patients with ST elevation vs non-ST elevation acute coronary syndrome. Circ J 2006; 70: 1372-8.

10. Irzmański R, Piechota $M$, Barylski $M$, et al. NT-proBNP in patients after acute coronary syndrome with ST segment elevation subjected to early posthospitalization cardiologic rehabilitation. Arch Med Sci 2006; 4: 262-7.

11. Baghdady Y, Kamel Y, Elwan A. N-Terminal pro-Brain natriuretic peptide in decompansated ventricular septal defect. Arch Med Sci 2008; 3: 376-82.

12. Lafontan M, Moro C, Sengenes C, Galitzky J, Crampes F, Berlan M. An unsuspected metabolic role for atrial natriuretic peptides: the control of lipolysis, lipid mobilization, and systemic nonesterified fatty acids levels in humans. Arterioscler Thromb Vasc Biol 2005; 25: 2032-42.

13. Mehra MR, Uber PA, Park MH, et al. Obesity and suppressed B-type natriuretic peptide levels in heart failure. J Am Coll Cardiol 2004; 43: 1590-5.

14. Messerli FH, Ventura HO, Reisin E, Dreslinski GR, Dunn FG, MacPhee AA. Borderline hypertension and obesity: two prehypertensive states with elevated cardiac output. Circulation 1982; 66: 55-60.

15. Hall C. Essential biochemistry and physiology of (NT-pro) BNP. Eur J Heart Fail 2004; 6: 257-60.

16. Moro C, Polak J, Richterova B, et al. Differential regulation of atrial natriuretic peptide- and adrenergic receptordependent lipolytic pathways in human adipose tissue. Metabolism 2005; 54: 122-31.

17. Kistorp C, Faber J, Galatius S, et al. Plasma adiponectin, body mass index, and mortality in patients with chronic heart failure. Circulation 2005; 112: 1756-62.

18. Birkenfeld AL, Boschmann M, Moro C, et al. Lipid mobilization with physiological atrial natriuretic peptide concentrations in humans. J Clin Endocrinol Metab 2005; 90: 3622-8.

19. Johnston Cl, Hodsman PG, Kohzuki M, Casley DJ, Fabris B, Phillips PA. Interaction between atrial natriuretic peptide and the renin angiotensin aldosterone system: endogenous antagonists. Am J Med 1989; 87: 24S-8S.

20. Prasad A, Quyyumi AA. Renin-angiotensin system and angiotensin receptor blockers in the metabolic syndrome. Circulation 2004; 110: 1507-12.

21. Buckley MG, Markandu ND, Miller MA, Sagnella GA, MacGregor GA. Plasma concentrations and comparisons of brain and atrial natriuretic peptide in normal subjects and in patients with essential hypertension. J Hum Hypertens 1993; 7: 245-50.

22. Hanley AJ, Festa A, D'Agostino RB Jr, et al. Metabolic and inflammation variable clusters and prediction of type 2 diabetes: factor analysis using directly measured insulin sensitivity. Diabetes 2004; 53: 1773-81.

23. Galasko GI, Lahiri A, Barnes SC, Collinson P, Senior R. What is the normal range for $\mathrm{N}$-terminal pro-brain natriuretic peptide? How well does this normal range screen for cardiovascular disease? Eur Heart J 2005; 26: 2269-76.

24. Wang TJ, Larson MG, Levy D, et al. Impact of age and sex on plasma natriuretic peptide levels in healthy adults. Am J Cardiol 2002; 90: 254-8.

25. Krauser DG, Lloyd-Jones DM, Chae CU, et al. Effect of body mass index on natriuretic peptide levels in patients with acute congestive heart failure: a ProBNP Investigation of Dyspnea in the Emergency Department (PRIDE) substudy. Am Heart J 2005; 149: 744-50. 